\title{
Orale Behandlungsregimes von Vorteil
}

_ Den Blutzuckerzielwert erreichen und kardiovaskuläres Risiko senken möglichst ohne Hypoglykämien: Das gehört zu den wesentlichen Zielen in der Therapie des Typ-2-Diabetes. Mit den modernen oralen Antidiabetika sind sie durchaus erreichbar.

Orale Antidiabetika oder schon früh Insulin? Daten zu diesen Fragen liefert aktuell eine nationale schwedische Beobachtungsstudie [Nyström T et al. Diabetes Obes Metab. 2017;19:831-41], die bei insgesamt 21.758 gematchten Patienten mit Typ-2-Diabetes DPP-4-Hemmer und SGLT-2-Inhibitoren mit Insulin unter Alltagsbedingungen verglich. Das Follow-up lag bei etwa eineinhalb Jahren.
Die Ergebnisse sprechen klar für das orale Therapieregime mit den neueren Antidiabetika: Die Gesamtmortalität lag unter oraler Therapie um 44\% niedriger als unter Insulin. Dabei zeigte der SGLT2-Hemmer Dapagliflozin (Forxiga) einen besseren Effekt im Vergleich $\mathrm{zu}$ dem DPP-4-Hemmer (-56\% versus $-41 \%)$. Die Häufigkeit tödlicher und nichttödlicher kardiovaskulärer Ereignisse wurden im Vergleich zu Insulin um 49\% beziehungsweise 13\% gesenkt.

Von Vorteil sind moderne orale Antidiabetika aber auch im Vergleich mit Sulfonylharnstoffen hinsichtlich des Hypoglykämierisikos, wie PD Jens Aberle, Hamburg-Eppendorf, anhand einer Ka- suistik erläuterte. Der kardiovaskulär vorbelastete Patient mit Typ-2-Diabetes, arterieller Hypertonie und einem Lateralwandinfarkt in der Anamnese war mit Metformin $2 \times 1.000 \mathrm{mg} / \mathrm{d}$ und Glimepirid $1 \times 2 \mathrm{mg} / \mathrm{d}$ eingestellt. Für Aberle war mit Blick auf das kardiovaskuläre Risiko und das Hypoglykämierisiko schnell klar: „Glimepirid muss raus.“ Wegen des „kranken Herzens“ des Patienten entschied er sich für einen Vertreter aus der Wirkstoffklasse der SGLT-2-Inhibitoren, deren günstige kardiovaskuläre Effekte belegt sind.

Dr. Beate Fessler

- Industriesymposium "Status \& Trends der patientenzentrischen Therapie des Typ-2-Diabetes. Hart aber fair diskutiert", DGIM 2017; Mannheim, Mai 2017 (Veranstalter: AstraZeneca)

\section{Rhinitis, Rhinosinusitis, Bronchitis}

\section{Zuerst Phytotherapeutika!}

— „Phytotherapeutika sind bei akuten Atemwegsinfektionen Antibiotika in vielen Bereichen überlegen“, betonte Dr. Katja Linke aus Viernheim. Dabei sei zu beachten: Qualität und Wirksamkeit eines Phytotherapeutikums hängen sowohl von der Zusammensetzung als auch von einem standardisierten Produktionsverfahren ab. Studiendaten zu einer bestimmten Wirkstoffkombination können nicht ohne weiteres auf ein ähnliches Präparat ohne entsprechende Daten übertragen werden. Auch bei Teemischungen sei es schwierig, geeignete Präparate zu bekommen, so die Allgemeinärztin.

\section{Verringerung des Hustens mit Thymian-Efeu-Kombination} In der S3-Leitlinie Husten (AWMF Nr. 053/013) werden aufgrund positiver randomisierter Studien Thymian-EfeuPräparate, Thymian-Primelwurzel-Präparate und Myrtol empfohlen. In der S3-Leitlinie ,akuter und chronischer Husten“ (AWMF Nr. 020/003) gibt es nur für definierte Extraktkombinationen aus Thymian/Efeu (Bronchipret ${ }^{\circledR}$ Kapseln und -Saft) bzw. aus Thymian/ Primel (Bronchipret ${ }^{\circledR}$-Pastillen) eine positive Empfehlung. Mit der Thy-mian-Efeu-Kombination wurde in einer placebokontrollierten Studie bei Patienten mit akuter Bronchitis die Zeit, bis eine Verringerung des Hustenscores um 50\% erreicht wurde, von 8 auf 6 Tage reduziert.

Kürzlich erschienen ist die neue S2kLeitlinie zur Rhinosinusitis (AWMF Nr. 017/049). Als evidenzbasierte Phytotherapeutika werden hier der patentierte

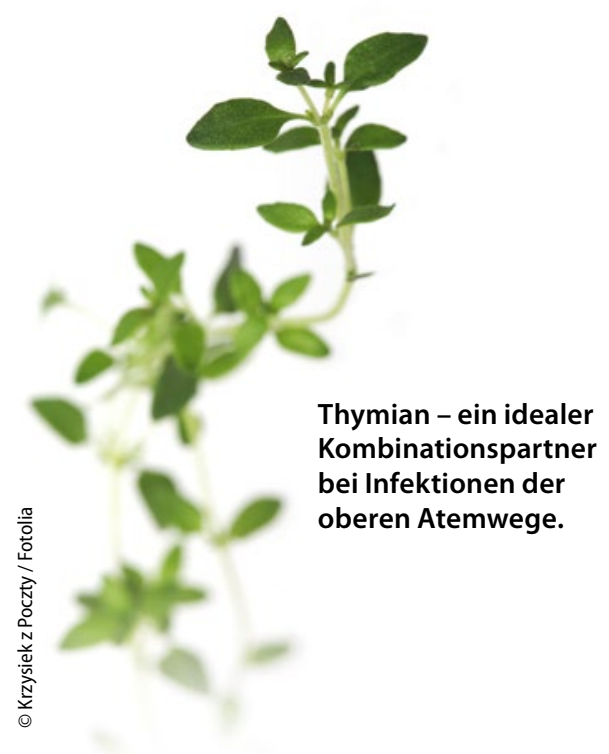

5-Pflanzen-Extrakt BNO1016 (Sinupret $^{\oplus}$ ) oder definierte Eukalyptus-Extrakte empfohlen.

\section{Roland Fath}

- 33. Seminarkongress Norddeutscher Hausärzte; Lüneburg, Mai 2017 